\title{
Wave Fields in Weyl Spaces and Conditions for the Existence of a Preferred Pseudo-Riemannian Structure
}

\author{
J. Audretsch ${ }^{1}$, F. Gähler ${ }^{2}$, and N. Straumann ${ }^{3}$ \\ 1 Fakultät für Physik der Universität Konstanz, D-7750 Konstanz, Federal Republic of Germany \\ 2 Institute for Theoretical Physics, ETH-Hönggerberg, CH-8093 Zürich, Switzerland \\ 3 Institute for Theoretical Physics of the University of Zürich, Schönberggasse 9, CH-8001 Zürich, \\ Switzerland
}

\begin{abstract}
Previous axiomatic approaches to general relativity which led to a Weylian structure of space-time are supplemented by a physical condition which implies the existence of a preferred pseudo-Riemannian structure. It is stipulated that the trajectories of the short wave limit of classical massive fields agree with the geodesics of the Weyl connection and it is shown that this is equivalent to the vanishing of the covariant derivative of a "mass function" of nontrivial Weyl type. This in turn is proven to be equivalent to the existence of a preferred metric of the conformal structure such that the Weyl connection is reducible to a connection of the bundle of orthonormal frames belonging to this distinguished metric.
\end{abstract}

\section{Introduction}

In the past, there have been several attempts to deduce the pseudo-Riemannian structure of general relativity theory from a few axioms. An interesting approach in this direction has been worked out by Ehlers, Pirani, and Schild [1], which starts with a set of events, $M$, and two families of subsets of $M$ which represent the collection of all (possible) light rays, and of all (possible) free fall world-lines of structureless test particles.

From a number of qualitative assumptions about light propagation and free fall which are mathematical idealizations of well-established facts and which appear to be minimal requirements of the local validity of special relativity, it has been shown by these authors that there is a unique Lorentzian conformal structure, (i.e. an equivalence class of pseudo-Riemannian metrics of Lorentzian signature) whose null geodesics are identical to the light rays. Moreover, the freely falling particles determine the affine geodesics (a geodesic path structure) of a class of projectively equivalent symmetric linear connections. The compatibility requirement that the null geodesics defined by the conformal structure 
belong to the ("deparametrized") geodesics of the distinguished class of connections leads to a Weyl structure of space-time.

One of the present authors (J. A.) has recently proposed an additional assumption [2], which implies in a natural and convincing manner the space-time structure of general relativity theory: If one requires that the trajectories of massive wave fields in the short wave limit coincide with the geodesics of the Weyl space, then this space is already a Lorentzian manifold, i.e., there is a distinguished metric of the conformal structure with respect to which lengths and times at different points can be compared in a path independent manner.

In this paper, we try to improve the technical treatment of ref. [2] by using the language of fiber bundles. The concepts and methods of fiber bundles provide a natural and convenient framework for a (global) discussion of the reduction of a Weyl space to a pseudo-Riemannian manifold, which is along the line of other examples of bundle reductions which appear in gauge theories (e.g. spontaneous symmetry breaking). We hope that the structural aspects of the problem will thereby be clarified.

Section 2 contains a discussion of the geometry of Weyl spaces. In Sect. 3 we introduce spin structures on Weyl spaces and formulate field equations for massive spinor fields. The Klein-Gordon theory is generalized to Weyl spaces in Sect. 4. In Sect. 5 we derive a necessary and sufficient condition that the trajectories of the WKB-limit agree with the geodesics of the Weyl connection. The geometrical significance of this condition will be elucidated in Sect. 6 and our conclusions will be summarized in Sect. 7.

\section{Geometry of Weyl Manifolds}

Let $M$ be a four-dimensional smooth manifold which is connected and paracompact.

A conformal structure on $M$ is an equivalence class $[\mathrm{g}]$ of conformally equivalent Lorentz metrics with signature $(+---)$. The pair $(M,[g])$ is called a conformal manifold. In a conformal manifold we can introduce the bundle of conformal frames, which are the linear frames consisting of pairwise orthogonal tangent vectors of equal length (relative to any $g \in[g]$ ). We assume that $M$ is space and time orientable and that an orientation is chosen. The set $W(M)$ of oriented conformal frames on $M$ can be regarded in an obvious manner as the total space of a principal fiber bundle $\pi: W(M) \rightarrow M$ whose structure group $G$ is the subgroup of the conformal group consisting of all nonzero multiples of homogeneous Lorentz transformations in $L_{+}^{\uparrow}$. Clearly, $G$ is isomorphic to $L_{+}^{\uparrow} \times \mathbb{R}_{+}$.

The principle bundle of (oriented) conformal frames, which we call the conformal bundle, is a reduction of the bundle $L(M)$ of linear frames. The canonical 1-form $\theta$ on $W(M)$ is just the restriction of the soldering form on $L(M)$. A Weyl connection is a connection $\omega$ on $W(M)$ with vanishing torsion form (i.e. $D^{\omega} \theta=0$, where $D^{\omega}$ is the exterior covariant derivative belonging to $\omega$ ). Since the connection form has values in the Lie algebra $\mathfrak{6}$ of $G$, i.e., in $\operatorname{so}(1,3) \oplus \mathbb{R}$, we can split $\omega$ uniquely

$$
\omega=\hat{\omega}+\varphi \cdot 1,
$$


where $\hat{\omega}$ has values in so $(1,3)$ and $\varphi$ is an $\mathbb{R}$-valued 1 -form. Thus, in matrix notation

$$
\hat{\omega}^{T} \eta+\eta \hat{\omega}=0, \omega^{T} \eta+\eta \omega=2 \varphi \eta,
$$

where $\eta=\operatorname{diag}(1,-1,-1,-1)$.

A Weyl connection can be considered as a torsion free linear connection, which is reducible to a connection in $W(M)$. The restriction of $\omega$ to any orthonormal frame bundle $O_{g}(M) \subset W(M), g \in[g]$, defines the Levi-Civita connection in $O_{g}(M)$.

Since the torsion vanishes, the first structure equation reads

$$
d \theta+\omega \wedge \theta=0 .
$$

The curvature $\Omega=D^{\omega} \omega$ is determined by the second structure equation

$$
\Omega=d \omega+\omega \wedge \omega,
$$

which can be written as

$$
\Omega=(d \hat{\omega}+\hat{\omega} \wedge \hat{\omega})+d \varphi \cdot 1 .
$$

A Weyl space is a conformal manifold together with a Weyl connection.

Matter fields, in particular tensor fields, are described by vector-valued tensorial forms of type $(\rho, V)$, where $\rho$ is a representation of the gauge group in the vector space $V$ (see, e.g., [3], Sect. II.5). (These tensorial forms can alternatively be regarded as sections of the associated bundle with typical fiber $V$.) In order not to overload notations and the degree of abstractness, we work in the following mostly with local expressions belonging to the local sections $\sigma_{\alpha}$ which are associated to local trivializations $T_{\alpha}: \pi^{-1}\left(U_{\alpha}\right) \rightarrow U_{\alpha} \times G$, where $\left\{U_{\alpha}\right\}$ is an open covering of $M$. The transition functions from $T_{\alpha}$ to $T_{\beta}$ are denoted by $f_{\alpha \beta}: U_{\alpha} \cap U_{\beta} \rightarrow G$.

The local representatives $\psi_{\alpha}=\sigma_{\alpha}^{*} \psi$ of a matter field $\psi$ of type $(\rho, V)$ transform as

$$
\psi_{\beta}=\rho\left(f_{\beta \alpha}\right) \psi_{\alpha} \text {. }
$$

In particular, for the curvature forms $\Omega_{\alpha}=\sigma_{\alpha}^{*} \Omega$, we have

$$
\Omega_{\beta}=\operatorname{Ad}\left(f_{\alpha \beta}^{-1}\right) \Omega_{\alpha} .
$$

The local representatives of the exterior covariant derivative $D \psi$ transform also according to the rule (2.5). Explicit formulas are

$$
(D \psi)_{\alpha}=d \psi_{\alpha}+\rho_{*}\left(\omega_{\alpha}\right) \wedge \psi_{\alpha},
$$

where $\rho_{*}$ is the induced representation of the Lie algebra $\mathfrak{G}$ of $G$.

The frames $\sigma(x)=\left\{e_{\mu}(x)\right\}$ of a local section $\sigma: U \rightarrow W(U)$ are dual to the components $\theta^{\mu}$ of $\sigma^{*} \theta$,

$$
\theta^{\mu}\left(e_{v}\right)=\delta_{v}^{\mu} .
$$

For any metric $g \in[g]$ we can choose local sections such that the frames $\left\{e_{\mu}(x)\right\}$ are orthonormal with respect to $g$,

$$
g=\eta_{\mu \nu} \theta^{\mu} \otimes \theta^{v}
$$

The general formula (2.7) shows that the components of $D g$ relative to the basis 
$\left\{\theta^{\mu}\right\}$ are

$$
(D g)_{\mu \nu}=d \eta_{\mu \nu}-\omega_{\mu}^{\lambda} \eta_{\lambda \nu}-\omega_{\nu}^{\lambda} \eta_{\mu \lambda}=-2 \varphi \eta_{\mu \nu} .
$$

In other words,

$$
D g+F(g) \otimes g=0,
$$

where $F(g)$ is a 1 -form locally given by $F(g)=2 \sigma^{*} \varphi$, if $\sigma$ is chosen such that (2.9) holds. From (2.10), one finds easily that

$$
F\left(e^{\lambda} g\right)=F(g)-d \lambda \text {. }
$$

Sometimes the existence of a map $F:[g] \rightarrow \Lambda^{1}(M)$ satisfying $(2.11)$ is taken as the definition of a Weyl structure [4].

The map $F$ defines the transport of a metric along any curve $\gamma:[0,1] \rightarrow M$. Let $g_{p}$ be a metric on $T_{p} M, p=\gamma(0)$, where $g \in[g]$. Then we define the transported metric $\tau_{\gamma}\left(g_{p}\right)$ on $T_{q} M, q=\gamma(1)$, by

$$
\tau_{\gamma}\left(g_{p}\right)=\exp \left[\int_{\gamma} F(g)\right] g_{q} .
$$

One sees immediately from (2.11) that, for a given $g_{p}, \tau_{\gamma}\left(g_{p}\right)$ is independent of the representative $g$, but depends in general on the path $\gamma$ which connects $p$ with $q$. Thus the comparison of lengths of vectors at $p$ and $q$ is path dependent.

If $\tau_{t}$ denotes the transport of metrics from $p$ to $\gamma(t)$, one finds easily that

$$
\lim _{t \downarrow 0} \frac{1}{t}\left(\tau_{t}^{-1} g_{\gamma(t)}-g_{p}\right)=-F(g)(\dot{\gamma}(0)) g_{p},
$$

and thus with (2.10)

$$
\left(\nabla_{\gamma(0)} g\right)_{p}=\lim _{t \downarrow 0} \frac{1}{t}\left(\tau_{t}^{-1} g_{\gamma(t)}-g_{p}\right)
$$

This formula shows that $\tau_{t}$ agrees with the parallel transport of $g$ belonging to the given Weyl connection $\omega$.

Since locally $F(g)=2 \sigma^{*} \varphi$, we see from (2.12) and Stokes' theorem that the parallel transport of metrics is locally path independent iff $d \varphi=0$.

The representations $(\rho, V)$ of the group $G$ have the form

$$
\rho: \lambda \Lambda \mapsto \lambda^{w} \hat{\rho}(\Lambda), \lambda>0, \Lambda \in L_{+}^{\uparrow},
$$

where $\hat{\rho}$ is a representation of $L_{+}^{\uparrow}$ and $w$ is any real number, called the weight of the representation $\rho$. $V$-valued differential forms that transform with the representation (2.14) in Eq. (2.5) are said to have weight $w$. The space $V$ will always be assumed to have a metric $h$ (not necessarily positive definite) with respect to which $\hat{\rho}$ is orthogonal.

Consider two $V$-valued $k$-forms $\alpha, \beta$ which transform with the same representation $\hat{\rho}$ relative to $L_{+}^{\dagger}$, but have in general different weights $w(\alpha)$ and $w(\beta)$. For each section $\sigma$ we choose $g \in[g]$ such that it has the form (2.9) relative to $\sigma$. Then we can define a natural scalar product

$$
\langle\alpha, \beta\rangle=g\left(\alpha^{a}, \beta^{b}\right) h\left(v_{a}, v_{b}\right),
$$


where $v_{a}$ is a basis of $V, \alpha=\alpha^{a} v_{a}, \beta=\beta^{b} v_{b}$, and $g\left(\alpha^{a}, \beta^{b}\right)$ is the usual scalar product for differential forms:

$$
g\left(\alpha^{a}, \beta^{b}\right) \mu=\alpha^{a} \wedge * \beta^{b} .
$$

Here $\mu$ is the volume form and $*$ the Hodge-operator belonging to $g$.

The scalar product (2.15) depends on the section $\sigma$ and transforms with weight $w(\alpha)+w(\beta)-2 k$ (but is invariant relative to $L_{+}^{\uparrow}$ ). Note also that $w\left({ }^{*} \beta\right)=w(\beta)+$ $4-2 k$.

For the construction of Lagrangians, it is important that $\langle\alpha, \beta\rangle$ has weight zero (i.e., is independent of the section) if

$$
w(\alpha)+w(\beta)-2 k+4=0 .
$$

Consider now a $k$-form $\alpha$ and a $(k-1)$-form $\beta$ with compact support contained in an open set $U$. If $w(\alpha)$ and $w(\beta)$ satisfy $(2.17),\langle\alpha, D \beta\rangle \mu$ is gauge invariant and the following formula holds:

$$
\int_{U}\langle\alpha, D \beta\rangle \mu=\int_{U}\left\langle\delta^{\omega} \alpha, \beta\right\rangle \mu .
$$

Here $\delta^{\omega}$ denotes the covariant codifferential defined by $\delta^{\omega}=* D^{\omega} *$. (For general dimensions and other signatures of $[g]$, one has to add a sign factor.) For the proof of (2.18) we use first of all

$$
\int_{U}\langle\alpha, d \beta\rangle \mu=\int_{U}\langle\delta \alpha, \beta\rangle \mu
$$

where $\delta$ is the ordinary codifferential $\delta=* d *$. From (2.7) and (2.1) we have

$$
D \beta=d \beta+\hat{\rho}_{*}(\hat{\omega}) \wedge \beta+w(\beta) \varphi \wedge \beta .
$$

Using well-known properties of the $*$-operation, the invariance of the metric $h$ with respect to the representation $\hat{\rho}$, and $w(* \alpha)=-w(\beta)$ (which is implied by (2.17)), one shows easily that the contributions of the last two terms in (2.20) can be transformed such that (2.18) holds.

\section{Spin Structures and Dirac Equation on Weyl Manifolds}

There is a natural extension of the universal covering homomorphism $\Lambda$ : $\operatorname{SL}(2, \mathbb{C}) \rightarrow L_{+}^{\uparrow}$ to a homomorphism $\tilde{\Lambda}: \tilde{G} \equiv \operatorname{SL}(2, \mathbb{C}) \times \mathbb{R}_{+} \rightarrow G$ given by $\tilde{\Lambda}(\lambda A)=$ $\lambda \Lambda(A)$, where $A \in \operatorname{SL}(2, \mathbb{C}), \lambda \in \mathbb{R}_{+}$.

A spin structure over the Weyl bundle consists of a principle fiber bundle $\tilde{\pi}$ : $\tilde{W}(M) \rightarrow M$ with structure group $\widetilde{G}$ and a bundle homomorphism $p: \tilde{W}(M) \rightarrow W(M)$ such that the corresponding homomorphism $\widetilde{G} \rightarrow G$ agrees with $\tilde{\Lambda}$. We assume that such a spin structure exists. (The existence question is essentially identical to the one in general relativity and so the results of Geroch [5] apply here too.)

Since the Lie algebras $\widetilde{\mathfrak{F}}$ and $\mathfrak{G}$ of $\widetilde{G}$ and $G$ are isomorphic, there is for any Weyl connection $\omega$ of $W(M)$ a unique connection $\tilde{\omega}$ of $\tilde{W}(M)$ such that $p$ maps horizontal subspaces onto horizontal subspaces. Clearly,

$$
\tilde{\omega}=\tilde{\Lambda}_{*}^{-1} \circ p^{*} \omega
$$

where $\tilde{\Lambda}_{*}: \widetilde{\mathfrak{G}} \rightarrow \mathfrak{G}$ is the isomorphism induced by $\tilde{\Lambda}$. 
A Dirac spinor field is a $\mathbb{C}^{4}$-valued tensoriel function $\psi: \tilde{W}(M) \rightarrow \mathbb{C}^{4}$ belonging to the representation

$$
\rho(\lambda A)=\lambda^{-3 / 2}\left(\begin{array}{ll}
A & 0 \\
0 & A^{*-1}
\end{array}\right) \equiv \lambda^{-3 / 2} \hat{\rho}(A) .
$$

The weight $w(\psi)=-3 / 2$ is natural because spinors have dimension [length $]^{-3 / 2}$.

According to the general rules we have, relative to a section, explicitly

$$
D \psi=d \psi+\rho_{*}(\tilde{\omega}) \psi=d \psi+\frac{1}{4} \omega_{\alpha \beta} \sigma^{\alpha \beta} \psi+w(\psi) \varphi \psi,
$$

where

$$
\sigma^{\alpha \beta}=\frac{1}{2}\left[\gamma^{\alpha}, \gamma^{\beta}\right]
$$

The Lagrangian of a Dirac field is generalized in an obvious manner; relative to a section,

$$
\mathscr{L}=\left\{\frac{1}{2}\left(\bar{\psi} i \gamma^{\lambda} D_{\lambda} \psi+\text { h.c. }\right)-m \bar{\psi} \psi\right\} \mu(g) .
$$

This is gauge invariant if the weight of the mass function $m$ is - as expected from dimensional reasons - equal to -1 .

The Euler-Lagrange equation of (3.4) is Dirac's equation

$$
-i \gamma^{\lambda} D_{\lambda} \psi+m \psi=0 \text {. }
$$

For a formal derivation of (3.5) one needs (2.18) and $\delta^{\omega} \theta=0$, which is a direct consequence of the vanishing of torsion.

Equation (3.5) implies that the current

$$
j^{\lambda}=\bar{\psi} \gamma^{\lambda} \psi
$$

is conserved

$$
D_{\lambda} j^{\lambda}=0 \text {. }
$$

For this it is important to note that $D \gamma^{\mu}=0$, since $\gamma^{\mu}$ are constant matrices (independent of the section).

\section{Klein Gordon Fields in Weyl Spaces}

Scalar fields have dimension [mass] and thus we assign the weight -1 to such fields in Weyl spaces.

The Lagrangian density for a minimally coupled complex scalar field $\phi$ is

$$
\begin{aligned}
\mathscr{L} & =\frac{1}{2}\left[\eta^{\alpha \beta}\left(D_{\alpha} \phi\right)^{*} D_{\beta} \phi-m^{2} \phi^{*} \phi\right] \mu(g) \\
& =\frac{1}{2}\left[\langle D \phi, D \phi\rangle-m^{2} \phi^{*} \phi\right] \mu(g),
\end{aligned}
$$

and is obviously gauge invariant. We could add curvature terms without changing our main conclusions.

The Euler-Lagrange equation is

$$
\delta^{\omega} D \phi-m^{2} \phi=0 .
$$


This follows again from (2.18). Equivalently, we can write

$$
\left(\eta^{\alpha \beta} D_{\alpha} D_{\beta}+m^{2}\right) \phi=0 .
$$

The current

$$
j^{\lambda}=\frac{i}{2}\left[\phi^{*} D^{\lambda} \phi-\left(D^{\lambda} \phi\right)^{*} \phi\right]
$$

is covariantly conserved

$$
D_{\lambda} j^{\lambda}=0
$$

\section{WKB-Limit for Wave Fields in Weyl-Spaces}

In this section we study the short wave limit for the unquantized Dirac and KleinGordon fields, and investigate under which conditions the currents belonging to these wave fields are in lowest order geodesic (relative to a Weyl connection).

\section{(a) Dirac Fields}

In the short wave limit we can make the usual WKB-ansatz

$$
\psi=e^{i S / \hbar}\left[\psi_{0}+\frac{\hbar}{i} \psi_{1}+\cdots\right] .
$$

If we introduce the representation (5.1) into the Dirac equation (3.5), we obtain for the coefficient of $\hbar^{0}$ (writing $d S=\partial_{\mu} S \theta^{\mu}$ )

and the coefficient of $\hbar$ gives

$$
\left(\gamma^{\mu} \partial_{\mu} S+m\right) \psi_{0}=0
$$

$$
\left(\gamma^{\mu} \partial_{\mu} S+m\right) \psi_{1}+\gamma^{\mu} D_{\mu} \psi_{0}=0 .
$$

The homogeneous equations (5.2) are consistent with $\psi_{0} \neq 0$ if

$$
\eta^{\alpha \beta} \partial_{\alpha} S \partial_{\beta} S=\langle d S, d S\rangle=m^{2},
$$

as can be seen by operating on the left of (5.2) with $\left(\gamma^{v} \partial_{v} S-m\right)$. Equation (5.4) is the Hamilton-Jacobi equation.

Our main conclusion will not depend on (5.3), but let us note the following consequence of this equation. Taking the adjoint of (5.2) and multiplying the result with $\psi_{1}$ gives $\bar{\psi}_{0}\left(\gamma^{\mu} \partial_{\mu} S+m\right) \psi_{1}=0$, where we have used the fact that $S$ is real. Now we can apply Eq. (5.3) and find

$$
\bar{\psi}_{0} \gamma^{\mu} D_{\mu} \psi_{0}=0
$$

From this equation one deduces immediately the conservation law of the zeroth order current

$$
D_{\mu}\left(\bar{\psi}_{0} \gamma^{\mu} \psi_{0}\right)=0
$$

For this current we find from (5.2),

$$
j_{0}^{\mu}=\bar{\psi}_{0} \gamma^{\mu} \psi_{0}=\bar{\psi}_{0} \gamma^{\mu}\left(-\frac{1}{m} \gamma^{v} \partial_{v} S\right) \psi_{0}=-\frac{1}{m} \bar{\psi}_{0} \gamma^{\nu} \partial_{v} S \gamma^{\mu} \psi_{0},
$$


or, taking half of the sum of the last two expressions,

$$
j_{0}^{\mu}=-\frac{1}{m} \bar{\psi}_{0} \psi_{0} \partial^{\mu} S
$$

Now we determine $\nabla_{j_{0}} j_{0}$ :

$$
j_{0}^{\lambda} D_{\lambda} j_{0}^{\mu}=j_{0}^{\lambda}\left[\frac{1}{m^{2}}\left(D_{\lambda} m\right) \bar{\psi}_{0} \psi_{0} \partial^{\mu} S-\frac{2}{m} \bar{\psi}_{0}\left(D_{\lambda} \psi_{0}\right) \partial^{\mu} S-\frac{1}{m} \bar{\psi}_{0} \psi_{0} D_{\lambda} \partial^{\mu} S\right] .
$$

The first two terms on the right-hand side are by (5.7) proportional to $j_{0}^{\mu}$. In the last term we use

$$
D_{\lambda} \partial_{\mu} S=D_{\mu} \partial_{\lambda} S
$$

which follows from the general formula, $D^{2} \psi=\rho_{*}(\Omega) \wedge \psi$, for tensorial forms of type $\rho$, and the vanishing of the torsion. Indeed, since $S$ is a scalar of weight zero we have

$$
\begin{aligned}
D^{2} S=0 & =D\left(\partial_{\mu} S \theta^{\mu}\right)=D_{\lambda}\left(\partial_{\mu} S\right) \theta^{\lambda} \wedge \theta^{\mu}+\partial_{\mu} S D \theta^{\mu} \\
& =\frac{1}{2}\left(D_{\lambda} \partial_{\mu}-D_{\mu} \partial_{\lambda}\right) \theta^{\lambda} \wedge \theta^{\mu} .
\end{aligned}
$$

We note also that

$$
D \eta^{\mu \nu}=D \eta_{\mu \nu}=0 \text {, }
$$

because $\eta^{\mu v}$ has weight zero and thus $D^{\omega} \eta_{\mu v}=D^{\omega} \eta_{\mu v}=0$.

Using (5.9) and (5.10) we can write for the last term in (5.8):

$$
-j_{0}^{\lambda} \frac{1}{m} \bar{\psi}_{0} \psi_{0} D_{\lambda} \partial^{\mu} S=-\frac{1}{m} \bar{\psi}_{0} \psi_{0} D^{\mu}\left(\partial_{\lambda} S\right) j_{0}^{\lambda} .
$$

This is proportional to $D^{\mu} m$, because the derivative of the Hamilton-Jacobi equation (5.4) gives

$$
m D^{\mu} m=\eta^{\alpha \beta}\left(D^{\mu} \partial_{\alpha} S\right) \partial_{\beta} S=D^{\mu}\left(\partial_{\lambda} S\right) \partial^{\lambda} S .
$$

Using this in (5.11) we finally get

$$
j_{0}^{\lambda} D_{\lambda} j_{0}^{\mu}=\frac{1}{m}\left(\bar{\psi}_{0} \psi_{0}\right)^{2} D^{\mu} m+\text { terms proportional to } j_{0}^{\mu} .
$$

This shows that $j_{0}^{\mu}$ is always geodesic iff

$$
D m=0 .
$$

(b) Klein-Gordon Fields

Again we get in zeroth order the Hamilton-Jacobi equation and thus (5.12). The lowest order of the current (4.4) is

$$
j_{0}^{\mu}=\left(\phi_{0}^{*} \phi_{0}\right) \partial^{\mu} S
$$


From this and (5.12) we find

$$
\begin{aligned}
j_{0}^{\lambda} D_{\lambda} j_{0}^{\mu} & =2\left(\phi_{0}^{*} D_{\lambda} \phi_{0}\right) \partial^{\lambda} S j_{0}^{\mu}+\left(\phi_{0}^{*} \phi_{0}\right)^{2} m D^{\mu} m \\
& =2\left\langle\phi_{0} d S, D \phi_{0}\right\rangle j_{0}^{\mu}+\left(\phi_{0}^{*} \phi_{0}\right)^{2} m D^{\mu} m,
\end{aligned}
$$

and we are led to the same conclusion (5.14).

\section{Discussion of $D m=0$}

We have shown that the WKB-limit leads to geodesic particle trajectories if and only if $D m=0$. This condition can be discussed in various ways.

Relative to a section $\sigma$ we have

$$
D\left(\sigma^{*} m\right)=d\left(\sigma^{*} m\right)-\sigma^{*}(\varphi) \sigma^{*}(m)=0,
$$

because $m$ is a Lorentz scalar and has weight -1 . Thus $\sigma^{*}(\varphi)=d \ln \left(\sigma^{*} m\right)$, and hence $\varphi$ is closed,

$$
d \varphi=0 .
$$

Consequently, the $\mathbb{R}$-component of the curvature in $\left(2.4^{\prime}\right)$ vanishes,

Furthermore,

$$
\Omega=d \hat{\omega}+\hat{\omega} \wedge \hat{\omega} \in \operatorname{so}(1,3) .
$$

$$
d F(g)=0
$$

for every $g$, since $F(g)$ is locally the pull-back of $\varphi$ by an appropriate section map. From (2.12) we see with Stokes' theorem that the metric transport is locally pathindependent.

In bundle language we can argue as follows. $D m=0$ implies that $m$ is constant along every horizontal curve. Thus the set of points $W(p)$ in $W(M)$ that can be joined with $p$ with a horizontal curve consists of frames with the same mass. By the reduction theorem, $W(p)$ is a reduced sub-bundle of $W(M)$ whose structure group is the holonomy group $\phi(p)$ with reference point $p$, and the connection $\omega$ in $W(M)$ is reducible to a connection in $W(p)$ (see, e.g., [3], Sect. II.7). Clearly, $\phi(p)$ is contained in $L_{+}^{\uparrow}$, because all frames in the fiber of $W(p)$ through $p$ have the same length relative to any $g \in[g]$.

Furthermore, by a theorem of Ambrose and Singer ([3], Sect. II.8) the Lie algebra of $\phi(p)$ is equal to the sub-space of $(\mathfrak{5}$ (Lie algebra of $G$ ) spanned by all elements of the form $\Omega_{q}(X, Y)$, where $q \in W(p)$ and $X$ and $Y$ are arbitrary (horizontal) vectors at $q$. Since $\phi(p) \subset L_{+}^{\uparrow}$, we conclude that $\Omega$ takes values in so $(1,3)$, which shows again that $d \varphi=0$.

Let $U$ be a simply connected open neighborhood of any point $x \in M$ and consider the induced connection in $W \mid U=\pi^{-1}(U)$. From (6.4) we know that there exists in $U$ a function $\lambda$ such that $F(g)=d \lambda$ in $U$, where $g$ is a given metric in $[g]$. Then by (2.11) $F\left(e^{\lambda} g\right)=0$, and hence (2.10) implies $D\left(e^{\lambda} g\right)=0$. Thus the connection is metrical relative to $\hat{g}=e^{\lambda} g$. This means that relative to any orthonormal tetrad (with respect to $\hat{g}$ ) the connection form $\omega$ is so(1,3)-valued. Hence the Weyl connection $\omega$ in $W \mid U$ is reducible to the orthonormal bundle $O_{\hat{g}}(U)$. 
We show now that the restriction to simply connected neighborhoods is not necessary and that there exists a $\hat{g} \in[g]$ such that $\omega$ is reducible globally to $O_{\hat{g}}(M)$.

For proving this we introduce the principle bundle with total space $\mathscr{M}=\left\{g_{x}: g \in[g], x \in M\right\}$, projection $\pi: \mathscr{M} \rightarrow M$ given by $\pi\left(g_{x}\right)=x$, and structure group $\mathbb{R}_{+}$operating as $g_{x} \mapsto \lambda g_{x}$.

There is a natural bundle homomorphism $f$ from $W(M)$ onto $\mathscr{M}(M)$ which sends a frame at $x \in M$ to the metric at $x$ with respect to which the frame is orthonormal. Since $m$ is a Lorentz scalar, but has nonvanishing weight, $m$ projects to a function $m^{\prime}$ on $\mathscr{M}$ such that $m=f^{*} m^{\prime}$. Furthermore, to each connection $\omega$ in $W(M)$ there is a unique connection $\omega^{\prime}$ in $\mathscr{M}(M)$ such that horizontal subspaces are mapped into horizontal subspaces by $f$ (see [3], Sect. II.6). The condition $D^{\omega} m=0$ goes over to $D^{\omega^{\prime}} m^{\prime}=0$.

The crucial point is now that the bundle $\mathscr{M}(M)$ is trivial. In fact, any $g \in[g]$ defines a trivialization $T_{g}: \mathscr{M} \rightarrow M \times \mathbb{R}_{+}$by $T_{g}\left(g_{x}^{\prime}\right)=(x, \lambda)$, where $\lambda$ is determined by $g_{x}^{\prime}=\lambda g_{x}$.

Repeating the argument which led to (6.4) we see now that $F(g)$ is (globally) exact for every $g$ and thus there exists a $\hat{g} \in[g]$ with $D \hat{g}=0$ on $M$. Hence we conclude that the connection $\omega$ is reducible to $O_{\hat{g}}(M)$.

By the construction above, we see that $O_{\hat{g}}(M)=f^{-1}(\sigma(M))$ as a set, where $\sigma$ : $M \rightarrow \mathscr{M}(M)$ is a global section with constant mass $m_{0}$ (and $O_{\hat{g}}(M)$ consists of all points in $W(M)$ with mass $m_{0}$ !). Clearly, we stay within $O_{\hat{g}}(M)$ along horizontal curves, because $m$ remains constant. This shows again that $\omega$ is reducible to $O_{\hat{g}}(M)$.

\section{Concluding Remarks}

The previous discussion can easily be generalized to arbitrary spin by studying the short wave limit of the massive Fierz-Pauli equations on Weyl manifolds.

It should be clear that one obtains in all cases the Hamilton-Jacobi equation for the eikonal $S$. Furthermore, the gradient of $S$ is always proportional to the zeroth order particle current, i.e., tangent to the trajectories of the WKB-limit. As for the Dirac and Klein-Gordon fields this leads to $D m=0$.

We have shown that this condition implies the existence of a preferred metric $g$ in the conformal class $[g]$ such that the Weyl connection of the bundle belonging to $[g]$ is reducible to a connection in the bundle of orthonormal frames belonging to $g$.

In the language developed by Trautman [6] we could regard a mass function $m$ of a nontrivial Weyl type as a Higgs field, and the equation $D m=0$ as the condition for spontaneous symmetry breaking of the group $G$ down to $L_{+}^{\dagger}$. With these remarks, we would like to emphasize that our discussion of the reduction of a Weyl space to the pseudo-Riemannian structure of general relativity is analogous to the bundle reductions which occur in ordinary spontaneously broken gauge theories.

In his Banff lectures [7] Ehlers concludes the discussion of his work with Pirani and Schild [1] with the following remarks:

"It must be admitted that the motivation of (the vanishing of the 'distance curvature') is not very satisfactory; it is an extraneous element of the theory. It seems that a deeper understanding of it could come from a better analysis of the 
description of matter in a Weyl spacetime. It is quite possible that such an analysis (may be in terms of possible wave equations for massive particles) would show that a 'reasonable' description of matter is possible only if the Weyl space is, in fact, Riemannian."

We hope that our investigation has at least partially fulfilled the expectation expressed in these sentences.

Finally, we emphasize that the axiomatic scheme of [1] excludes from the very beginning a non-vanishing torsion. It should be clear that the conclusions of this paper also rest heavily on this assumption.

Acknowledgements. We thank the organizers and participants of the meeting on General Relativity on Schloss Ringberg (Sept. 1983) for critical and constructive remarks. Special thanks go to J. Ehlers for his interest in this work.

\section{References}

1. Ehlers, J., Pirani, F. A. E., Schild, A.: The geometry of free fall and light propagation. In: General Relativity, O'Raifeartaigh, L. (ed.). Oxford: Clarendon Press 1972

2. Audretsch, J.: Riemannian structure of space-time as a consequence of quantum mechanics. Phys. Rev. D27, 2872-2884 (1983)

3. Kobayashi, S., Nomizu, K.: Foundations of differential geometry, Vol. 1. New York: Interscience 1963

4. Folland, G. B. : Weyl manifolds. J. Diff. Geom. 4, 145-153(1970)

5. Geroch, R. : Spinor structures of space-times in general relativity I. J. Math. Phys. 9, 1739-1744 (1968)

6. Trautman, A. : Fibre bundles, gauge fields, and gravitation. In: General Relativity and Gravitation, Vol. 1, Held, A. (ed.). New York, London: Plenum Press 1980

7. Ehlers, J.: Survey of general relativity. In: Relativity, Astrophysics and Cosmology, Israel, W. (ed.). Dordrecht, Boston: Reidel 1973

Communicated by S. Hawking

Received November 23, 1983 
